\title{
Pediatric Deep Venous Thrombosis and Pulmonary Embolism: Can It Be Antiphospholipid Syndrome?
}

\section{Pediatrik Derin Ven Trombozu ve Pulmoner Emboli: Antifosfolipid Sendromu Olabilir mi?}

(D) Fatma Demir Yenigürbüz, (D) Hale Ören

Dokuz Eylül University Faculty of Medicine, Department of Pediatric Hematology, İzmir, Turkey

To the Editor,

In pediatric patients with deep venous thrombosis (DVT) and pulmonary embolism (PE), antiphospholipid syndrome (APS) should be considered early and efforts must be made to ensure timely diagnosis of this potentially life-threatening condition. Pediatric APS is an autoimmune disease characterized by vascular thrombosis and persistently positive antiphospholipid antibodies $[1,2,3,4,5]$. Primary APS is rarely seen in childhood [4]. A 14-year-old adolescent was admitted with complaints of left upper leg edema for 1 week. On physical examination, obesity, hypertension, and edema of the leg were present. Hyperlipidemia and D-dimer elevation were remarkable. Doppler ultrasonography showed DVT in his left femoral vein and abdominal computed tomography (CT) demonstrated iliac vein thrombosis (Figure 1). Since he had widespread DVT, thorax CT

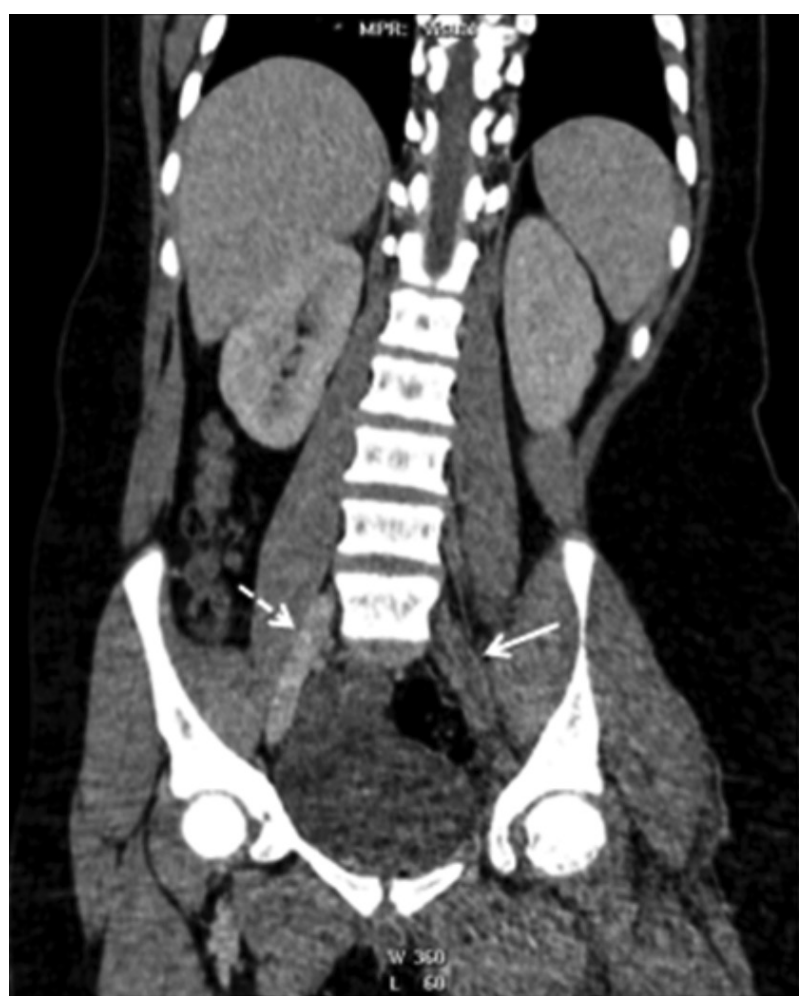

Figure 1. Abdominal computed tomography of the patient demonstrating iliac vein thrombosis (arrows). angiography was also performed without any clinical symptoms of PE and it demonstrated filling defects in the right pulmonary artery (Figure 2). Anticoagulation was given and complete recanalization was observed. A diet program was started. When thrombophilia risk factors were evaluated, there was no family history and the genetic thrombophilia panel was negative, LA was positive twice with an interval of 12 weeks (first sample was before treatment), and other APS antibodies were found negative. Systemic lupus erythematosus (SLE) and SLE-like diseases were excluded. The patient was diagnosed with primary APS. Metabolic syndrome was the additional thrombotic risk factor. Long-term anticoagulation therapy (lifetime) was given to the patient.

Keywords: Deep venous thrombosis, Pulmonary embolism, Antiphospholipid syndrome

Anahtar Sözcükler: Derin ven trombozu, Pulmoner emboli, Antifosfolipid sendromu

Informed Consent: Received.

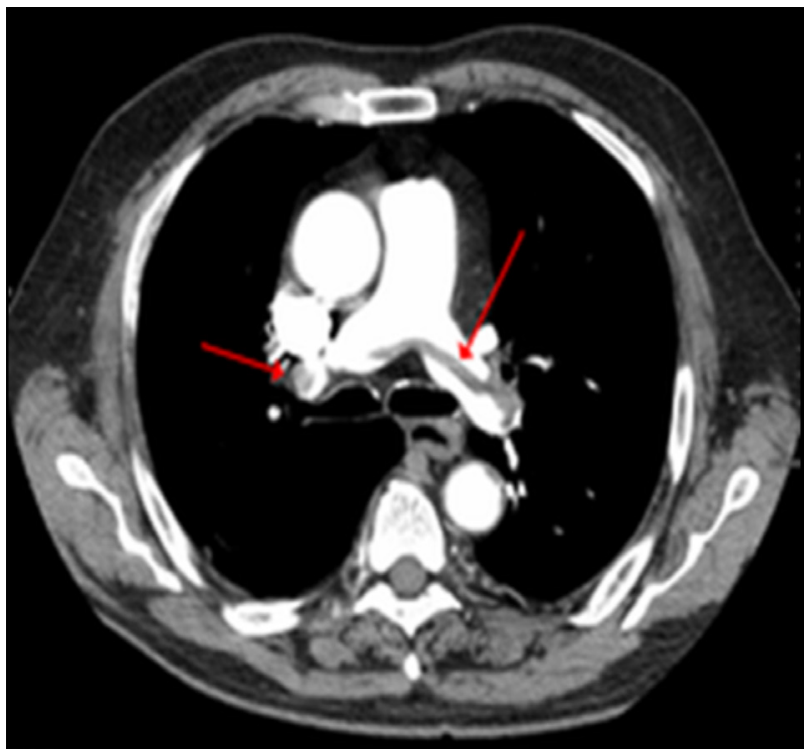

Figure 2. Thorax computed tomography angiography of the patient demonstrating filling defects in right pulmonary artery (arrows). 
Conflict of Interest: The authors of this paper have no conflicts of interest, including specific financial interests, relationships, and/or affiliations relevant to the subject matter or materials included.

\section{References}

1. Yang JY, Chan AK. Pediatric thrombophilia. Pediatr Clin North Am 2013;60:1443-1462.

2. Biss T. Pulmonary embolism in childhood: how can we be sure not to miss it? Arch Dis Child 2018:103:814-816.
3. Aguiar CL, Soybilgic A, Avcin T, Myones BL. Pediatric antiphospholipid syndrome. Curr Rheumatol Rep 2015;17:27.

4. Rumsey DG, Myones B, Massicotte P. Diagnosis and treatment of antiphospholipid syndrome in childhood: a review. Blood Cells Mol Dis 2017;67:34-40.

5. Groot N, de Graeff N, Avcin T, Bader-Meunier B, Dolezalova P, Feldman B, Kenet G, Koné-Paut I, Lahdenne P, Marks SD, McCann L, Pilkington CA, Ravelli A, van Royen-Kerkhof A, Uziel Y, Vastert SJ, Wulffraat NM, Ozen S, Brogan $P$, Kamphuis $S$, Beresford MW. European evidence-based recommendations for diagnosis and treatment of paediatric antiphospholipid syndrome: the SHARE initiative. Ann Rheum Dis 2017;76:1637-1641.

\title{
Pediatric Chronic Myeloid Leukemia Presenting in a Mixed Phenotypic Blast Crisis: A Rare Occurrence
}

\author{
Bir Karışık Fenotipik Blast Krizinde Pediatrik Kronik Myeloid Lösemi Sunumu: \\ Nadir Bir Durum
}

\author{
(D) Jenna Bhattacharya, (D) Richa Gupta \\ Maulana Azad Medical College, Department of Pathology, New Delhi, India
}

\section{To the Editor,}

Pediatric chronic myeloid leukemia (CML) comprises 3\% of childhood leukemia cases [1]. Similar to adults, most of the patients present in the chronic phase, but 5\% may present in a blast crisis (BC) [2]. Mixed phenotypic $\mathrm{BC}$ has rarely been reported in children [3]. A 10-year-old male presented with fever, fatigue, dull abdominal pain, and massive splenomegaly for 2 months. Complete blood count results were as follows: total leukocyte count (TLC), 544×109/L; hemoglobin, $8 \mathrm{~g} / \mathrm{dL}$; and platelet count, $80 \times 109 / \mathrm{L}$. Differential count on peripheral smear revealed the following: blasts - $25 \%$, promyelocytes - 3\%, myelocytes - 20\%, metamyelocytes - 10\%, eosinophils - 5\%, basophils - 4\%, monocytes - 2\%, lymphocytes - $16 \%$, and neutrophils $-15 \%$. No dysplasia was noted. The blasts had moderate cytoplasm and prominent nucleoli. On cytochemistry, these blasts were negative for myeloperoxidase and periodic acid-Schiff. Bone marrow aspirate revealed a hypercellular marrow with myeloid predominance (M:E ratio of 25:1) with $55 \%$ blasts. Megakaryocytes were adequate with some dwarf forms. Bone marrow biopsy was hypercellular with near total replacement of marrow spaces with sheets of blasts having vesicular nuclei and prominent nucleoli (Figure 1). Blasts were positive for CD34, anti-MPO, CD19, and CD20 (Figure 1) and negative for CD3. Considering the high TLC and peripheral blood and bone marrow picture, RT-PCR for $M-B C R-A B L 1$ was done, which confirmed the presence of a 210-kDa transcript. Considering the clinical presentation, the peripheral blood picture (basophilia, many myelocytes and metamyelocytes), and the 210-kDa BCR-ABL1 transcript, a diagnosis of mixed phenotypic $B C$ in CML was issued and treatment was initiated with imatinib. Subsequently, the patient improved with lowering of TLC and disappearance of blasts from the peripheral blood. However, molecular response in follow-up could not be determined due to economic constraints.

The incidence of progression to BC in adults is 10\% but the same is not well known in children [3]. BC is usually myeloid and rarely mixed phenotypic [4]. The biology of progression of pediatric CML to $B C$ is supposed to be similar to that of adults [2]. Accumulation of additional chromosomal anomalies in the proliferating clone, especially deletions in the $C D K N 2 A / B$ gene, deletions in the IKZF gene, and chromosomal aberrations associated with myelodysplasia, have been implicated with progression [5]. Mixed phenotypic BC in CML needs to be differentiated from de novo mixed phenotypic acute leukemia (MPAL). Differentiation may be difficult since MPAL can also show $M-B C R-A B L 1$ translocation [6]. The points in favor of 